\title{
Identidad cultural del Municipio de Sitionuevo mediada por la investigación como estrategia pedagógica ${ }^{1}$
}

\section{Cultural identity of the Municipality of Sitionuevo mediated by research as a pedagogical strategy}

\author{
DOI: http://dx.doi.org/10.17981/cultedusoc.9.3.2018.109
}

Artículo de investigación. Fecha de recepción: 15/06/2018. Fecha de aceptación: 27/11/2018

\author{
Julieth Rico-Yépez²; \\ Milena Roenes-Romero; Alejandro Salas-Meriño; Ivon Ramos-Feria; \\ Vilma Narváez-Gómez; Lila Mena-Rodríguez; Nancy Martínez-Castro; \\ Isabel Cabrera-Mejía; Ana San José-Fernández y Klemans Manga-Sarmiento ${ }^{3}$ \\ IED San José, sede ERM San Antonio (Colombia) \\ klemans-16@hotmail.com
}

Para citar este artículo:

Rico-Yépez, J., Roenes-Romero, M., Salas-Meriño, A., Ramos-Feria, I., Narváez-Gómez, V., Mena-Rodríguez, L., Martínez-Castro, N., Cabrera-Mejía, I., San José-Fernández, A. y Manga-Sarmiento, K. (2018). Identidad cultural del Municipio de Sitionuevo mediada por la investigación como estrategia pedagógica. Cultura. Educación y Sociedad 9(3), 919-928. DOI: http://dx.doi.org/10.17981/cultedusoc.9.3.2018.109

\section{Resumen}

La identidad cultural es aquella que constituye las características, culturales, gastronómicas, tecnológicas y artísticas propias de una región. Actualmente muchas de las tradiciones culturales se han dejado de reproducir, puesto que las generaciones de jóvenes se interesan por otro tipo de actividades, sin embargo, conocer la cultura hace parte de la construcción de identidad. Por tal motivo se realizó la presente investigación, cuyo objetivo fue emplear la IEP para fomentar la identidad cultural de los habitantes del municipio de Sitio Nuevo. Entre las técnicas de recolección empleadas se destacan la observación participante y no participante y las entrevistas en profundidad, las cuales fueron aplicadas a 90 (noventa) estudiantes de la institución Educativa San José, del municipio en mención. Los resultados obtenidos permitieron establecer estrategias de intervención efectivas para el uso en las asignaturas de la institución y para ser divulgadas en la comunidad en general.

Palabras clave: Identidad cultural, mediación investigación como estrategia pedagógica.

\section{Abstract}

The cultural identity is that which constitutes the characteristics, cultural, gastronomic, technological and artistic typical of a region, nowadays many of the cultural traditions have stopped reproducing since the generations of young people are interested in other types of activities, however, knowing the culture is part of the construction of identity. For this reason, the study aimed to promote the cultural identity of the Municipality of Sitio Nuevo mediated by the Research as an IEP Pedagogical Strategy. It was guided by the methodological guidelines proposed by the IEP, using information gathering techniques such as blogs and reflective journals. The participants were ninety (90) students from the San José Educational Institution, from the Municipality of Sitio Nuevo, Magdalena. The results showed the social appropriation of knowledge by students in the presentation of research projects at the institutional fair.

Keywords: Cultural identity, research mediation as a pedagogical strategy.

1 Este artículo ha sido derivado del Programa de Fortalecimiento de la Cultura Ciudadana y Democrática CT+I a través de la IEP
apoyada en TIC en el Departamento de Magdalena: CICLON

2 Líder Grupo de investigación "Juventud Antorcha".

3 Docentes de la Institución Educativa Distrital San José; Sede ERM San Antonio.

- The author; licensee Universidad de la Costa - CUC.

Cultura, Educación y Sociedad vol. 9 no. 3, pp. 919-928. Diciembre, 2018

Barranquilla. ISSN 2389-7724 Online 


\section{Introducción}

El concepto de identidad cultural puede ser entendido desde los fenómenos sociales transcurridos a lo largo del tiempo, en las dimensiones económica, humana, social y por supuesto, personal (Molano, 2007).

A su vez, la cultura desde el ámbito antropológico, se define como una construcción social, basados en la idea que los componentes biológicos no son determinantes del comportamiento humano, sino que má bien se configuran mediante sistemas simbólicos, que crean, producen y transmiten formas adecuadas de relaciones entre hombres y mujeres bajo la forma de reglas normativas, las cuales producen una forma de adaptación y utilización del ambiente que involucra la producción de conocimientos y técnicas, expresados como comportamientos estandarizados que son enseñados y transformados por cada generación (Durham, 2014; Durán y Parra, 2014).

El pensamiento sobre territorio e identidad cultural no está marcado por la idea de volver a los métodos usados en el pasado, si no que se tiene en cuenta que este es un proceso permanente de transformaciones, que proviene de las relaciones sociales, lo que indica relaciones de poder (Flores, 2007). En el uso de la identidad cultural como motor para el desarrollo de un territorio resulta particularmente útil conocer el origen y el contenido de las palabras y el significado de estas para una posterior interpretación, así como las normas y definiciones, que se han logrado establecer a través de consensos internacionales (Molano, 2006).

La simultaneidad del compromiso con los lugares de origen y los de destino ha producido formas de vida comunitaria con configuraciones territoriales y culturales novedosas, si un grupo de personas están acostumbradas a cierto estilo de vida, el cambiar este por otro puede resultar difícil, pero siempre será una bonita experiencia que muchas personas buscan seguir (Velasco, 1998).

La Constitución Política de 1991 establece que el patrimonio cultural es un punto de referencia que, al generar la construcción de lazos sociales, vínculos de cohesión, en los distintos niveles de gobierno, permite un desarrollo social, democrático y económico (Uribe,2006).

El tema de identidad, está siendo vigorosamente debatido en la teoría social, el argumento central es que las antiguas identidades que estabilizaron el mundo durante tanto tiempo, hoy en día ya no tienen ese mismo valor, y esto da origen a otras nuevas y fragmenta al individuo moderno, a esto se le llama "crisis de identidad" (De pablos, 1999).

La construcción de la identidad cultural se propone como una de las claves para afrontar el reto que supone un cambio educativo, esto hace referencia a cambiar la forma de pensar y ver el mundo, pues aprender culturas y costumbres nuevas es una forma de profundizar, en el marco de los denominados estudios culturales (Aponte, 2003).

A través de la vida el ser humano construye en el medio, acciones que van transcurriendo, como elementos que cambian, modifican o hacen permanecer lo que se desea, pasando por períodos creativos o de transición, creando costumbres e ideas nuevas, cada una de las costumbres son las que crean la identidad cultural. La comunicación es la fuente fundamental para el desarrollo, para las formas de vida del ser humano y a lo largo de la historia se han constituido en la base para la identidad cultural, estos dos aspectos involucran obligatoriamente procesos de comunicación y son tan cotidianos dentro de la sociedad (Ortiz, 2015). El espacio relacionado con el tema de del discurso social, el de la cultura y la comunicación es, consecuentemente, espacio de modelación social y, por ende, espacio de disputas 
y negociaciones, conflictos y acuerdos del orden del sentido (Mata, 1994).

A través de la búsqueda de conocimiento y de las experiencias se constituye el saber conocer, el saber hacer y el saber ser. Todo esto con el fin de alcanzar lo que verdaderamente una identidad, el pasado, el presente y el futuro que se avecina (Masonyi, 1982). Un proceso que afecta el desarrollo de identidad sobre la base de experiencias tempranas marcadas por la vivencia de estar "al margen de" y la consolidación de representaciones sociales que les ubica culturalmente en un espacio social con características particulares, es conducta delictiva que presentan algunos jóvenes que viven en condiciones de pobreza, lo que lleva a un proceso de inadaptación social (Zambrano, Pérez, 2004).

En el municipio de Sitio nuevo, Magdalena; la cultura representa una importante fuente de experiencias significativas para sus habitantes, ya que en ella se fundamentan muchos de los valores y costumbres de las personas que allí habitan; pero estas no se reconocen así mismas y no reconocen sus talentos, que pueden a través de sus acciones y la puesta en práctica de las mismas engrandecer su futuro, hacerlo más prometedor para ellos y para sus familias (Díaz, 1998).

Teniendo en cuenta interrogantes que han surgido de algunos miembros de la vereda San Antonio pertenecientes al municipio de Sitionuevo, Magdalena, en cuanto a la falta de proyección, de las diferentes danzas, muestras artesanales, gastronómicas que identifiquen no solamente el Municipio, Corregimiento y Veredas, al igual que los otros aledaños al rio magdalena, han surgido ideas para responder a dichos interrogantes. Desarrollar dicha cultura no es, sin embargo, una tarea fácil, pues requiere la convergencia de políticas de control con políticas de carácter educativo y durante el desarrollo del Programa de Fortalecimiento de la
Cultura Ciudadana y Democrática CT+I a través de la IEP apoyada en TIC en el Departamento de Magdalena: CICLON, está planteado mediante la siguiente pregunta de investigación:

¿Cómo se fomentaría la identidad cultural de Sitio Nuevo mediante la implementación de la IEP?

Investigación como estrategia pedagógica apoyada en TIC

La investigación como estrategia pedagógica se observa como una herramienta educativa que permite generar visiones innovadoras en el proceso de enseñanzaaprendizaje desde una perspectiva crítica. Generando una reconfiguración del saber escolar, lo cual se debe convertir en una oportunidad para ir más allá de la modernización de la educación e intentar transformar las relaciones de poder que controlan y dominan con propuestas pedagógicas y metodológicas inclusivas, que muestren diseños concretos y humanizados, basados en el aprendizaje contextual, generando practicas pedagógicas que permiten la exploración, indagación y análisis de la información, que permite contrastar la teoría con la práctica (Mejía y Manjarrés, 2010).

En ese sentido, considerar la investigación como estrategia pedagógica implica recrear un espacio de inclusión, donde se respete y se viva la diversidad haciendo evidentes los canales de la creatividad del docente, para convertir las preguntas de los grupos de aprendizaje colaborativo conformados por niños, niñas y adolescentes en una investigación, llevándolos a tomar un rol activo en el proceso de aprendizaje mediante la construcción de su propia conocimiento, potenciando así, las habilidades y destrezas de los estudiantes, convirtiendo la educación en un vehículo de capacitación, de oportunidades y de recreación. Estos elementos permiten fortalecer adicionalmente los procesos 
de responsabilidad social educativa frente a la proyección de las instituciones en el marco de sus PEI (Sanz y Crissien, 2012; Camargo, 2015).

Desde la metodología propuesta por la IEP los contenidos curriculares se convierten en problemas, donde el docente a partir de las preguntas planteadas por los estudiantes diseña unas trayectorias de indagación, así, los avances y desarrollos de los estudiantes se determinan por la ampliación de estructura previa que se manifiesta en lenguaje, raciocinios y conocimientos. Ese descubrimiento no es necesariamente autónomo, sino que se hace guiado por el profesor, quien se encarga de planificar los ejercicios y los procedimientos pare el fin buscado, (Pozo y Gómez, 2006). Autores como Pinto y Misas (2014) destacan el rol del docente en la formación del estudiante desde su mediación para el desarrollo autónomo, estimulación temprana y generación de opacidades para la toma de decisiones.

La propuesta metodológica se fundamenta en valores que buscan desarrollar capacidades de cognitivas, afectivas, valorativas y de acción. En ese sentido, pensar la investigación desde las corrientes educativas criticas significa una lucha teóricopráctica por la manera como sus supuestos sobre el conocimiento, la ciencia, su epistemología, la cultura, lo humano y los grupos sociales enmarcan una acción que ha sido señalada como objetiva, y que no solo ha construido una forma de ella, sino que también ha ayudado a generar formas de poder que en la sociedad han servido para el control y la gestación de desigualdades (Ortega, Peñuela y López, 2009).

En resumen, el docente desde la IEP parte de la pregunta de sentido común de los estudiantes, la redirecciona a la perspectiva de la educación, reconociendo la existencia de saberes comunes o elaborados, asociando los conocimientos disciplinares con las experiencias socioculturales, promoviendo así la apropiación practica desde el pensamiento crítico buscando transformar contextos, culturas, epistemologías, y redirigir sus escenarios de poder para construir subjetividades y ciudadanías (Mariño, 2010). Con esta postura coinciden autores como Pino y Urrego (2013) y Moreno (2015) cuando asignan al contexto social importancia preponderante en los procesos formativos del individuo.

De otra parte, según diversos autores las tecnologías de la información y la comunicación (TICs), permiten desarrollar competencias sociales, que incluso se ha utilizado para trabajar en instituciones educativas con deterioro de la convivencia escolar como factores asociados al desempeño en estudiantes de básica primaria con experiencia de desplazamiento forzado (Avendaño, Cortés, Guerrero, 2015; Pinto, Cortés y Alfaro, 2017).

Así mismo, las TIC son tecnologías para almacenar, recuperar, procesar y comunicar la información. Existe una variedad de recursos electrónicos que se encuadran dentro del concepto de TIC como; televisión, teléfonos, videos, ordenadores, entre otros.

Para realizar el proceso de implementación de las TIC al contexto educativo es necesario tener en cuenta el nivel de madures digital que presentan los usuarios y para esto se debe diseñar un modelo eficaz que permita medir dicho nivel de forma inicial, donde los sujetos observados participen plenamente, asumiendo el ejercicio a partir de la influencia de las TIC en el contexto contemporáneo (Rozo, et al, 2016).

Con las artes y las TIC como ejes canalizadores de todos estos conocimientos y aprendizajes estamos más que acampando en un puerto seguro, ellas son la ventana para mostrar y expandir al mundo como entes formadores de cultura e identidad tanto dentro de la escuela, como en el municipio y alrededor del mundo que se conectara con cada persona desde todas las perspectivas, puestas en marcha con las TIC. 


\section{Metodología}

\section{Diseño}

La investigación estuvo guiada bajo los lineamientos metodológicos de la Investigación como Estrategia Pedagógica IEP. El eje de la propuesta consiste en tomar los saberes propios de la cultura y la sociedad para asociarlos a las formas establecidas de conocimiento, es decir, una forma de integrar la teoría, a los aprendizajes cotidianos de las experiencias, por ello, la IEP plantea la creación de grupos infantiles y juveniles para generar la unidad y relación de saber y conocimiento como partes complementarias, a través de una propuesta metodológica que realiza el reconocimiento social de los actores, los cuales a través de la negociación cultural de sus preguntas estructuran las diferentes concepciones, las cuales podrán tramitar reconociendo la visibilidad de múltiples métodos investigativos, en coherencia con el tipo de problema.

Por ello, el proceso reconoce la investigación como una forma de cambiar concepciones, realidades y el entorno de los participantes, y con ello, una manera de situarse crítica y éticamente en el mundo cercano y mediato. Autores como García y Arrieta (2016) concuerdan en la necesidad de que en el ciclo didáctico se apliquen estrategias innovadoras para desarrollar el pensamiento crítico, la cognición y la metacognición. Esta postura complementa la necesidad de generar comunidades de aprendizaje colaborativo que compromete a sus comunidades para construir una cultura ciudadana en ciencia tecnología e innovación. (Mejía y Manjarrés, 2010).

\section{Participantes}

La unidad de análisis del estudio, estuvo conformada por noventa (90) estudiantes de la Institución Educativa Departamental San José, sede ERM San José del Rio, distribuidos en los cursos; octavo, noveno y décimo, con edades que oscilaron entre los catorce (14) y dieciséis (16) años (Tabla 1).

TABLA 1

Distribución de los participantes

\begin{tabular}{cc}
\hline Grado & $\begin{array}{c}\text { Número de } \\
\text { Estudiantes }\end{array}$ \\
\hline Octavo & 22 \\
Noveno & 24 \\
Decimo & 44 \\
TOTAL & 90 \\
\hline
\end{tabular}

Fuente: Elaboración propia

\section{Técnicas e instrumentos}

Luego de realizar los recorridos de las trayectorias de indagación, se escogieron como técnicas de recolección de información la observación participante y no participante y las entrevistas en profundidad, los cuales permiten tomar una información detallada y contextualizada de la experiencia investigativa tal cual como la viven los actores que participan en el proceso mismo.

\section{Procedimiento}

A partir de los recorridos de las trayectorias de indagación, basados en la integración de la IEP apoyada en TIC al aula, articulado al plan de estudios, desde las diferentes áreas se estructuraron temáticas relacionadas con la identidad cultural, de acuerdo a los diferentes momentos:

Momento (1): Conformación de los grupos de investigación.

Momento (2): integración de la temática mediante la IEP al aula de clases, se integraron grupos estudiantiles para seleccionar problemáticas relacionadas con la identidad cultural. Los estudiantes se plantearon preguntas y con el acompañamiento del docente realizaron el proceso de indagación mediante herramientas virtuales para luego realizar el proceso de construcción de sus productos. 
Momento (3): análisis de los resultados de la implementación de la estrategia a través del diario de campo y entrevistas.

Momento (4): socialización de los resultados de las indagaciones de estudiantes en una feria institucional dirigida por los docentes a cargo.

Momento (5): propagación y divulgación de los resultados.

\section{Resultados}

Los principales hallazgos del estudio fueron las diversas propuestas de investigación generadas por los estudiantes quienes observaron durante el proceso la importancia de rescatar y mantener la identidad de su pueblo, utilizando prácticas educativas innovadoras como la investigación como una estrategia pedagógica se contribuyó al trabajo de cada uno de los maestros con relación una nueva forma de impartir la enseñanza, y a los estudiantes como beneficiarios directos en su proceso de aprendizaje y formación a nivel académico, personal y profesional, lo que permite que tengan nuevas visiones y fortalezcan las habilidades con las que ya cuentan para desenvolverse en la vida cotidiana.

Para la Institución Educativa Departamental San José, sede ERM San José del Rio fue importante rescatar mediante la elaboración del proyecto todos estos antecedentes culturales que son el fundamento de su propia identidad, a través de muestras artísticas y gastronómicas las cuales deben proyectar al municipio no solo a nivel regional sino también nacional e internacional, todos esos saberes, vivencias y competencias laborales mediante la investigación cultural de las zonas ribereñas, donde los actores principales son los estudiantes.

A continuación, se presenta la sistematización de los resultados partiendo de los momentos metodológicos establecidos a través del diseño de las trayectorias de indagación propuestas en la ruta metodológica de la IEP. (Ver tabla 2).

TABLA 2

Sistematización de los resultados de la investigación

\begin{tabular}{lcc}
\hline $\begin{array}{c}\text { Categoría de } \\
\text { investigación }\end{array}$ & $\begin{array}{c}\text { Momento } \\
\text { metodológico }\end{array}$ & Actividad realizada \\
\hline
\end{tabular}

Durante esta fase, se conformaron 5 grupos de investigación por cada Momento 1 grado de la institución, los cuales

\begin{tabular}{|c|c|c|}
\hline \multirow{3}{*}{$\begin{array}{l}\text { Identidad } \\
\text { cultural del } \\
\text { Municipio. }\end{array}$} & Momento 2 & $\begin{array}{l}\text { - Planteamiento de preguntas. } \\
\text { - Planteamiento de problemas de investigación. } \\
\text { - Búsqueda en internet. } \\
\text { - Consulta a docentes. } \\
\text { - Entrevistas a la comunidad. }\end{array}$ \\
\hline & Momento 3 & $\begin{array}{l}\text { - Sistematización de la teoría en fichas bibliográficas. } \\
\text { - Análisis de las entrevistas. }\end{array}$ \\
\hline & $\begin{array}{l}\text { Momento } 4 \\
\text { y } 5\end{array}$ & $\begin{array}{l}\text { Apropiación social del conocimiento mediante estrategias de } \\
\text { divulgación del saber en la institución educativa, donde se presentaron } \\
\text { los resultados de las investigaciones y se realizaron muestras } \\
\text { representativas de la cultura del municipio. }\end{array}$ \\
\hline
\end{tabular}

Fuente: elaboración propia. 
De esta manera se observa cómo la integración de la investigación como estrategia pedagógica apoyada en las TIC, se convierte en una metodología educativa que permite generar conocimientos a través de un proceso de aprendizaje colaborativo; concebida como una nueva forma de trasmitir el conocimiento dejando atrás los paradigmas tradicionales de enseñanza, propiciando de esta manera una partica situada, significativa y contextualizada que dinamiza los procesos curriculares, posibilitando la participación, el diálogo, la reflexión, en pro de la transformación del entorno desde cambios significativos, basados en problemáticas cotidianas de su comunidad, partiendo del proceso de implementación de la IEP al aula se realizó la sistematización de la ruta metodológica (Figura 1).
La figura anterior, muestra la forma en la que los maestros y maestras interiorizaron el proceso de integración curricular de la IEP, observándolo como un proceso cíclico que conlleva a un aprendizaje constante basado en la adquisición de experiencias científicas, contextualizadas, donde el dialogo de saber toma un papel importante en el desarrollo de conocimiento.

\section{Discusión}

Los resultados anteriormente expuestos frente a la identidad cultural se sustentan con los planteamientos de Durham (2014), quien explica la cultura desde el ámbito antropológico, se define como una construcción social, basados en la idea

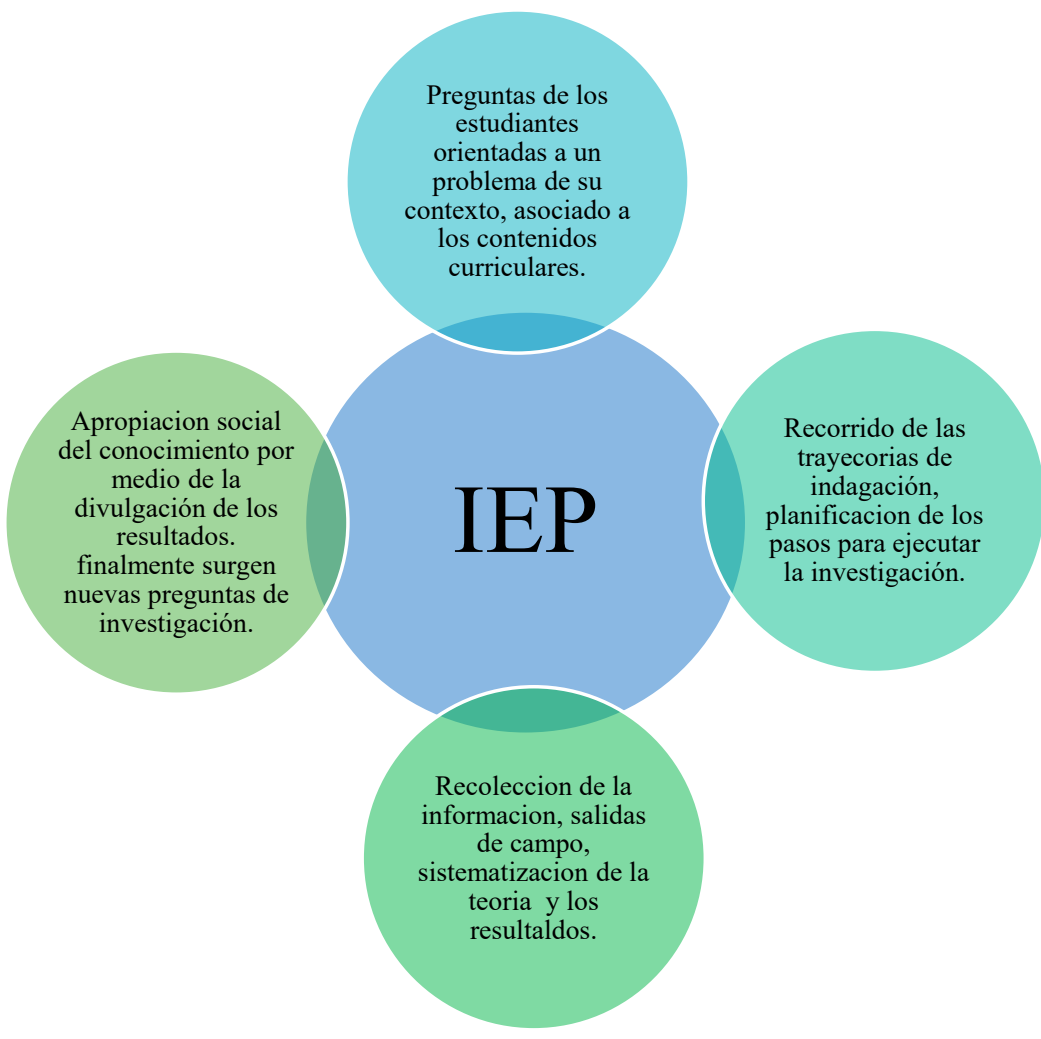

Figura 1. Sistematización de la ruta metodológica de la IEP

Fuente: elaboración propia. 
que los componentes biológicos no son determinantes del comportamiento humano, por tal razón se hace necesario realizar una organización de la conducta colectiva, mediante sistemas simbólicos, que crean, producen y transmiten formas adecuadas de relaciones entre hombres y mujeres bajo la forma de reglas normativas, se produce una forma de adaptación y utilización del ambiente que involucra la producción de conocimientos y técnicas, expresados como comportamientos estandarizados que son enseñados y transformados por cada generación. Observando que los estudiantes encontraron la identidad cultural de su pueblo basados en las historias de sus ancestros y sintieron la necesidad de rescatar las costumbres por medio de muestras, gastronómicas, folclóricas y artesanales.

Por su parte en lo que respecta con el proceso de integración de la IEP al aula de clases, los resultados se apoyan en los planteamientos de Mejía y Manjarrés (2010), quienes definen la investigación como estrategia pedagógica se observa como una herramienta educativa que permite generar visiones innovadoras en el proceso de enseñanza-aprendizaje desde una perspectiva crítica. Generando una reconfiguración del saber escolar, lo cual se debe convertir en una oportunidad para ir más allá de la modernización de la educación e intentar transformar las relaciones de poder que controlan y dominan con propuestas pedagógicas y metodológicas inclusivas, que muestren diseños concretos y humanizados, basados en el aprendizaje contextual, generando practicas pedagógicas que permiten la exploración, indagación y análisis de la información, que permite contrastar la teoría con la práctica. Lo cual se observó mediante la sistematización realizada sobre la ruta metodológica de la IEP.
Finalmente, a manera de recomendación es muy relevante que los espacios generados dentro de los programas y horarios académicos para la realización de las actividades diseñadas se mantengan, y así convertir las prácticas culturales en la herramienta de descubrimiento de próximas investigaciones y que la investigación sea considerada una práctica permanente en el currículo.

\section{Referencias}

Acevedo, C. (2017). Las tiendas de barrio desde la economía institucional. International Journal of Management Sciences and Operations Research, 2(1), 30-37. Recuperado de http://ijmsoridi. com/index.php/ijmsor/article/view/85

Aponte, G. (2003). Paisaje e identidad cultural. Revista Tabula Rasa, 1(1). 153164.

Araque, F. y Suárez, O. (2017). Equidad Ética-Jurídica de la Ciencia, para la Emancipación del Conocimiento y los Saberes. JURÍDICAS CUC, 13(1). 97120. DOI: http://dx.doi. org/10.17981/ juridcuc.13.1.2017.5

Avendaño, I., Cortés, O., y Guerrero, H, (2015). Competencias sociales y tecnologías de la información y la comunicación como factores asociados al desempeño en estudiantes de básica primaria con experiencia de desplazamiento forzado. Divers: Perspect. Psicol. 11(1)13-36.

Belloch (2012). Las Tecnologías de la Información y Comunicación en el aprendizaje. Recuperado de https://www. uv.es/bellochc/pedagogia/EVA1.pdf

Camargo, C. (2015). La investigación como estrategia pedagógica en la Guajira, desde una perspectiva de la inclusividad y diversidad como nuevo paradigma para el desarrollo de habilidades, destrezas y competencias. Educación y ciudad, (29), 149-162. 
Carvajal, P. (2013). El reconocimiento de derechos a la comunidad LGBTI. Jurídicas CUC, 9(1), 123 - 141.

Cortázar, J. (2006). Estrategias educativas para el desarrollo de una cultura tributaria en América Latina. Experiencias y líneas de acción. Revista del CLAD.

De Pablos, J. (1999). Las nuevas tecnologías y la construcción de la identidad cultural (el cambio educativo para el siglo XXI). Bordón. Revista de pedagogía, 51(4), 417-433.

Díaz-Couder, E. (1998). Diversidad cultural y educación en Iberoamérica. América, 17(1). 1-15.

Durán, S. y Parra, M. (2014). Diversidad Cultural para promover el desarrollo de habilidades sociales en educación superior. Cultura, Educación y Sociedad, 5(1), 55-67.

Durham, E. (2014). Cultura, patrimonio, preservación. Alteridades, (16), 131-136.

Flores, M. (2007). La identidad cultural del territorio como base de una estrategia de desarrollo sostenible. Revista Opera, 7(7). 35-54.

García, H. y Arrieta, A. (2016). Potencialización del Pensamiento Crítico a través de la Hipotemetacomprensión Textual (Hmt). Cultura Educación y Sociedad, 7(2), 54-71. Recuperado de https://revistascientificas.cuc.edu.co/culturaeducacionysociedad/article/view/1102

Grossberg, L. (2003). Identidad y estudios culturales:¿no hay nada más que eso? En, S. Hall, Cuestiones de identidad cultural, (148-180). Madrid: Ediciones Amorrortu.

Hall, S. (2003). Introducción: ¿ Quién necesita identidad? En, S. Hall, Cuestiones de identidad cultural, (13-39). Madrid: Amorrortu Editores.

Hall, S. (2010). Sin garantías: trayectorias $y$ problemáticas en estudios culturales. Popayán: Envion editores.
Hernández, J.J. (2007). Toma de decisiones públicas desde las perspectivas del proceso tecnocrático y la participación ciudadana: caso venezolano. Revista Venezolana de Gerencia, 12(40). 553571.

Hernández, D. y Escobar, A. (2017). Modelo de contabilidad social como herramienta de gestión para la responsabilidad social empresarial. International Journal of Management Sciences and Operations Research, 2(1), 44-56. Recuperado de http://ijmsoridi.com/index.php/ijmsor/article/ view/86

Mariño, G. (2010). El diálogo en la educación de jóvenes y adultos. Dos propuestas pedagógicas para implementarlo. En, G. Mariño El taller dialógico / la recuperación de experiencias laborales. Bogotá, D.C.: OEI.

Mata, M. (1994). Nociones para pensar la comunicación y la cultura masiva. En, Espacio Curricular. Extensión Rural. Compendio Didáctico. Año 2018 (5662). Universidad Nacional de Córdoba. Recuperado de http://agro.unc.edu. ar/ extrural/Compendio.pdf

Mejía, M. y Manjarrés, M. (2010). La Investigación como Estrategia Pedagógica. Bogotá, D.C.: Ondas-Colciencias.

Meza, A., Arrieta, M. y Noli S. (2018). Análisis de la conciliación extrajudicial civil en la Costa Atlántica colombiana. JURÍDICAS CUC, 14(1). 187-210. DOI: http://dx.doi. org/10.17981/juridcuc.14.1.2018.9

Molano, L., y Lucía, O. (2007). Identidad cultural un concepto que evoluciona. Opera, (7). 69-84.

Molano, O. (2006). La identidad cultural, uno de los detonantes del desarrollo territorial. Revista Territorios con identidad cultural. Recuperado de http://goo.gl/LYkKl 
Moreno, G. (2015). Una Aproximación al concepto de Observatorio Social. Cultura Educación y Sociedad, 6(1). 93-108. Recuperado de https://revistascientificas.cuc.edu.co/culturaeducacionysocie$\mathrm{dad} / \mathrm{article/view/758}$

Mosonyi, E. (1982). Identidad nacional y culturas populares. Caracas: La Enseñanza Viva

Ortega, P., Peñuela, D. y López, D. (2009). Sujetos y prácticas de la pedagogía critica. Bogotá, D.C.: El Búho.

Ortiz, C. y Guevara, J. (2015). La Educomunicación y la identidad cultural en los estudiantes de octavo, noveno y décimo año de educación básica de la unidad Educativa Tamboloma de la Parroquia Pilahuin de la Ciudad de Ambato provincia del Tungurahua. (Bachelor's thesis). Universidad Técnica de Ambato, Ecuador.

Pastrano, R. y Campuzano, L. (2013). La identidad nacional y su influencia en el respeto de la diversidad cultural en los estudiantes de $1^{\circ}$ a $10^{\circ}$ año general básica. (Bachelor's thesis). Universidad de Guayaquil. Ecuador.

Pinto, M. y Misas, M. (2014). La educación inicial y la educación preescolar: perspectivas de desarrollo en Colombia y su importancia en la configuración del mundo de los niños. Cultura Educación y Sociedad, 5(2). 119-140. Recuperado de https://revistascientificas.cuc.edu. co/culturaeducacionysociedad/article/ view/889

Pino, M. y Urrego, Y. (2013). La importancia de las funciones ejecutivas para el desarrollo de las competencias ciudadanas en el contexto educativo. Cultura Educación y Sociedad, 4(1). 9.20. Recuperado de https://revistascientificas. cuc.edu.co/culturaeducacionysociedad/ article/view/969
Pinto, A., Cortés, O. y Alfaro, C. (2017). Hacia la transformación de la práctica docente: modelo espiral de competencias TICTACTEP. Pixel-Bit, 51. 37-51.

Pozo, J. y Gómez, M. (2006). Aprender y enseñar ciencia. Madrid: Ediciones Morata.

Rozo, J., Martin, A., Fagua, A. y Ávila, Y. (2016). Estrategias de gamificación aplicadas al diagnóstico de la incorporación pedagógica de las TIC en una comunidad académica. Cultura Educación y Sociedad, 4(1). 55.74.

Santos, A. y Peña, O. (2017). ¿Qué piensan los estudiantes universitarios frente a la formación investigativa?. REDU. Revista de Docencia Universitaria, 15(2), 57-76.

Sanz, A. y Crissien, T. (2012). "Respondabilidad" en las instituciones de educación superior. Cultura, Educación y Sociedad. 3(1), 147-156.

Uribe, S. (2006). La identidad cultural y el desarrollo territorial rural, una aproximación desde Colombia. Revista Territorios con identidad cultural. Recuperado de http://recursos.salonesvirtuales.com/assets/bloques/ Soto_URIBE_desarrolloterritorialrural.pdf

Velasco, M. (1998). Identidad cultural y territorio: una reflexión en torno a las comunidades trasnacionales entre México y Estados Unidos. Región y Sociedad, 9(15). 104-130.

Zambrano, A. y Pérez-Luco Arenas, R. (2004). Construcción de identidad en jóvenes infractores de ley, una mirada desde la Psicología Cultural. Revista de Psicología, 13(1). 115-132. https:// w w w.doi.org/ $10.5354 / 0719$ 0581.2012 .17491 